\title{
Radio to X-rays properties of TeV blazars
}

\author{
Zulema Abraham ${ }^{1}$ and Tânia P. Dominici ${ }^{1}$ \\ ${ }^{1}$ Instituto de Astronomia, Geofísica e Ciências Atmosféricas, Universidade de São Paulo, Rua \\ do Matão 1226, Cidade Universitária, 05508-900, São Paulo, SP, Brazil
}

\begin{abstract}
The detection of very high energy emission in blazars with the new generation of $\mathrm{TeV}$ telescopes rises the question of the origin of this emission and its relation to that at other wavelengths. In this work we present contemporaneous optical and X-ray observations of two bright and close southern hemisphere blazars: PKS 2005-489 and PKS 2155-304, the latter detected at $\mathrm{TeV}$ energies. The $\mathrm{SED}$ between radio and X-rays is discussed and compared with that of other $\mathrm{TeV}$ blazars.
\end{abstract}

\section{Introduction}

Blazars, which include the Optical Violent Variable objects, the flat spectrum radio quasars and the BL Lac objects, have Spectral Energy Distributions (SED) that extends sometimes up to $\mathrm{TeV}$ energies. The BL Lac sources do not present spectral lines, implying that the non-thermal processes dominate the emission. They present compact radio structure, sometimes not resolved or with core-jet structure extending for a few mas which can be resolved into discrete components moving away from the core, with not very large velocities (Piner \& Edwards 2004). In this work we are trying to answer the following questions: are these properties intrinsic to the blazars, maybe related to the central black hole mass and rotation? Are they the effect of orientation, as in the Unified Model, forming very small angles with the line of sight? Are they the consequence of interactions of the relativistic jet with the local environment? For this we analyzed the SED from radio to X-rays of two nearby blazars, PKS 2005-489 and PKS 2155-304, the latter a well established source of $\mathrm{TeV}$ emission (Chadwick et al. 1999). We also searched for correlations between the optical and X-ray light curves of these objects.

\section{The SED}

We used optical data obtained at the Abrahão de Moraes Observatory (Valinhos, Brazil) and contemporaneous BeppoSax observations at X-rays to construct the SED, that can be see in Figure 1, complemented with NED infrared and radio data. A parabolic fit was applied to the data to estimate the synchrotron peak frequency.

The analysis of the SED indicates that in both sources the variability in the optical range has smaller amplitude than that found in X-rays. Moreover, smooth variation in the spectral index $\left(S \propto \nu^{-\alpha}\right)$ can explain very well the SEDs at all epochs. Radio emission does not follow the fit and is probably due to shocks.

\section{Comparison between optical and X-rays light curves}

We searched for correlations between our optical observations and the $1.5-12 \mathrm{keV}$ data obtained in the All Sky Monitor Project (ASM, http://xte.mit.edu). The Discrete Correlation Function (DCF, Edelson \& Krolik 1988) was used to quantify the correlations and time lags. For PKS 2005-489, a possible delay with the optical emission preceding 

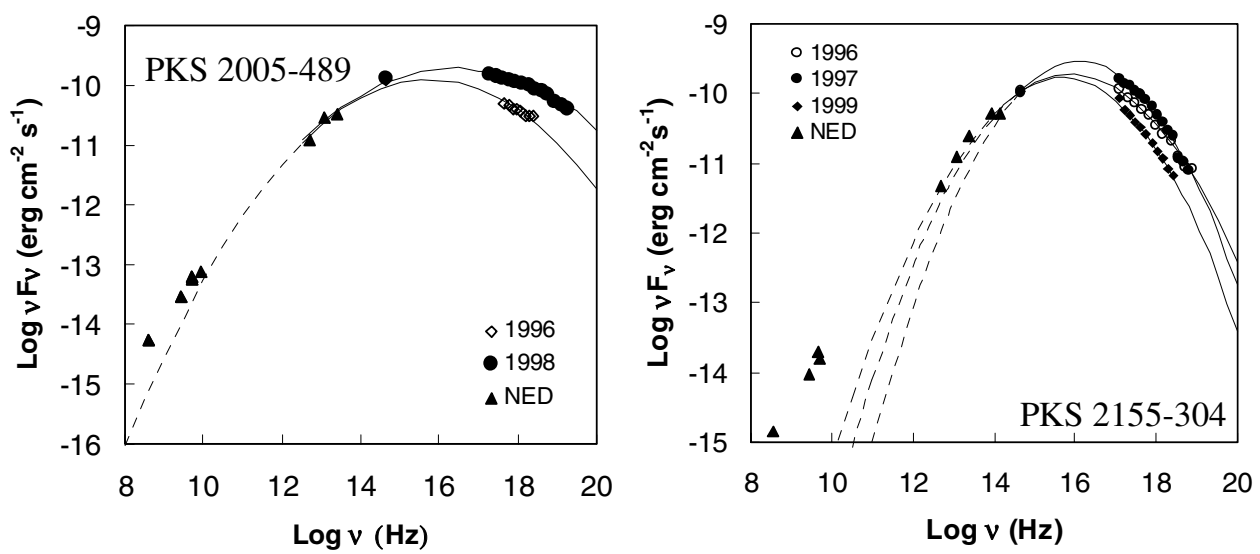

Figure 1. Spectral energy distribution (SED) for PKS 2005-489 (left) and PKS 2155-304.

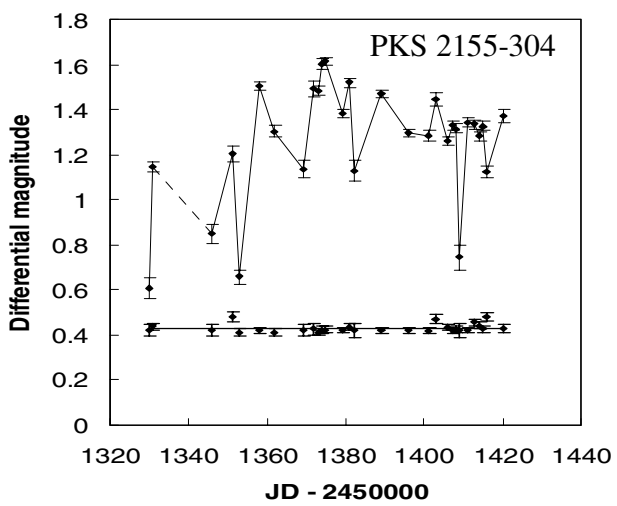

Figure 2. Upper curve: differential light curve of the BL Lac PKS 2155-304 in 1999. Lower curve: the control light curve.

the X-rays by 30 days was found in 1997, while in 1998 the X-rays preceded the optical emission by 10 days. Variability occurring first at X-rays can be explained as synchrotron emission, from perturbation propagating along the jet. X-ray after optical emission can be explained by Inverse Compton, with radio or IR photons.

Analyzing the 1999 optical light curve of PKS 2155-304, showed in Figure 2, we see at least two sharp dips. We interpreted them as eclipses of a region moving with relativistic velocity along a direction close to the line of sight by clouds of the BLR. These clouds could also provide the photons for the EC TeV emission.

\section{Acknowledgements}

This work is partially supported by Brazilians agencies FAPESP and CNPq. This research made use of the NASA/IPAC Extragalactic Database (NED).

\section{References}

Chadwich, P. M., et al. 1999, ApJ, 513, 161

Piner, B. G., \& Edwards, P. G. 2004, ApJ, 600, 115

Edelson, R. A., \& Krolik, J. H. 1988, ApJ, 333, 646

Dominici, T. P. 2003, PhD Thesis, IAG-USP

Dominici, T. P., Abraham, Z., Teixeira, R., \& Benevides-Soares, P. 2004, AJ, in press 